\title{
Pulmonary rehabilitation for cystic fibrosis: A narrative review of current literature
}

\author{
Evgenia I. Kalamara1, Evangelos T. Ballas ${ }^{2}$, Georgia Pitsiou ${ }^{1,3}$, Guergana Petrova ${ }^{2,4}$ \\ ${ }^{1}$ Respiratory Failure Unit, General Hospital of Thessaloniki “G. Papanikolaou”, Thessaloniki, Greece; ${ }^{2}$ Medical \\ University Sofia, Bulgaria; ${ }^{3}$ Medical school Aristotle university of Thessaloniki; ${ }^{4}$ Pediatric Clinic, University Hospital \\ Alexandrovska, Sofia, Bulgaria
}

\begin{abstract}
Pulmonary rehabilitation is a key component in cystic fibrosis care. This review summarizes the recent evidence in the area of pulmonary rehabilitation for cystic fibrosis in the form of questions and answers regarding interventions, indications, benefits and risks of pulmonary rehabilitation. Pulmonary rehabilitation includes airway clearance techniques, exercise training, education and behaviour change and can improve patients' exercise capacity, muscle strength, quality of life and nutritional status. Airway clearance techniques have beneficial effects for clearing mucous. Over the past years, evidence for the beneficial effects of exercise training on exercise capacity and
\end{abstract}

Correspondence: Evgenia I. Kalamara, Pavlou Mela 3, Chortiatis, 57010 Thessaloniki, Greece.

Tel.+30.6948107468. E-mail: gina.kal88@gmail.com

Key words: Pulmonary rehabilitation; cystic fibrosis; exercise capacity; lung function; quality of life; physiotherapy; airway clearance; exercise training.

Conflict of interest: The authors declare that they have no competing interests, and all authors confirm accuracy.

Contributions: All the authors made a substantive intellectual contribution, have read and approved the final version of the manuscript and agreed to be accountable for all aspects of the work.

Ethics approval: Not applicable.

Availability of data and materials: The data used to support the findings of this study are available from the corresponding author upon request.

Received for publication: 11 July 2020.

Accepted for publication: 22 December 2020.

${ }^{\circ}$ Copyright: the Author(s), 2021

Licensee PAGEPress, Italy

Monaldi Archives for Chest Disease 2021; 91:1501

doi: 10.4081/monaldi.2021.1501

This article is distributed under the terms of the Creative Commons Attribution Noncommercial License (by-nc 4.0) which permits any noncommercial use, distribution, and reproduction in any medium, provided the original author(s) and source are credited. overall lung health is growing. In cystic fibrosis, multiple factors result in reduced exercise capacity. All modalities of pulmonary rehabilitation should be offered to patients with cystic fibrosis, as the benefits in most cases outweigh the risks, though the optimal regimens need to be yet defined.

\section{Introduction}

Cystic fibrosis $(\mathrm{CF})$ is an autosomal recessive disease and one of the most common life-shortening condition affecting approximately 70,000 individuals worldwide [1], caused by mutations in the cystic fibrosis transmembrane conductance regulator (CFTR) gene. The CFTR protein is expressed in epithelial cells and has several functions, primarily serving as an ion channel. It regulates liquid volume on epithelial surfaces through chloride secretion and inhibition of sodium absorption.

Airway disease is hypothesized to be mainly a result of reduced airway surface liquid volume which subsequently leads to mucociliary dysfunction, increase of bacterial load, and chronic inflammation. The abnormal composition and secretion of mucus affects multiple organs and causes various complications, thus rendering $\mathrm{CF}$ a systemic disease. Therefore, $\mathrm{CF}$ management requires a multidisciplinary approach, preferably in the setting of a specialized center. The treatment should be individualized to the needs of each patient, and should aim at the management of airway infection and the development of an active lifestyle, in order to maintain good nutrition and exercise capacity [2].

Pulmonary rehabilitation is an intervention customized to each patient and includes airway clearance techniques, exercise training, education and behaviour change. Its target is the amelioration of the physical and psychological status of people with chronic respiratory disease and the promotion of long-term adoption of health- improving behaviour. It is acknowledged as an important adjunction to the treatment of many chronic respiratory diseases [3]. In patients with CF, pulmonary rehabilitation traditionally focused on airway clearance techniques. Nowadays the management also extends to other areas. Systematic exercise training and personalized rehabilitation programs are offered to CF patients [4], while new technologies are being gradually incorporated to the rehabilitation process during the past few years.

This review was conducted in order to map the recent research done in the area of pulmonary rehabilitation for cystic fibrosis and answer questions about its main aspects, as an aid for respiratory medicine physicians, general practitioners, physiotherapists or other clinicians involved in the care of CF patients. 


\section{Methods}

This literature review took place between March 2019 and September 2020. Our protocol was drafted using the Preferred Reporting Items for Systematic Reviews and Meta-analysis Protocols, with appropriate modifications for the purposes of a narrative review [5]. For this review all relevant information was identified using Embase, Cochrane Library, Science Direct, Scopus, PubMed databases and Google Scholar.

A literature search was performed. Search words were cystic fibrosis, pulmonary rehabilitation, exercise capacity, lung function, quality of life, physiotherapy, airway clearance, exercise training. Relevant peer-reviewed journal papers were included if they were published between the period of 2000 to 2020 and written in English. The papers could include subjects of various ages (children, adolescents, adults) and severity of disease. Papers were excluded if they did not fit into the conceptual framework of the study, for example papers focused on chronic respiratory conditions other than cystic fibrosis.

The selected literature included 23 Randomized control trials, 8 Cochrane Systematic Reviews, 7 guidelines, 7 clinical studies, 12 reviews, 1 systematic review, 2 other original articles.

\section{What interventions does pulmonary rehabilitation include?}

\section{Airway clearance techniques}

An integral part of pulmonary rehabilitation is the application of airway clearance techniques, that are used to enhance the mucociliary clearance system, in order to transport secretions proximally up the airways [6].

These include conventional chest physiotherapy (mainly involves postural drainage, percussion and vibration), active cycle of breathing techniques ( $A C B T$ ), which consist of breathing control exercises, thoracic expansion exercises and forced expirations, positive expiratory pressure (PEP) therapy, which is defined as breathing against a positive expiratory pressure using a mask or mouthpiece, autogenic drainage (three-level breathing sequence beginning at low lung volumes, followed by breathing at mid-lung volumes, followed by deep breathing, huff and coughing- it uses controlled breathing to achieve the highest possible airflow in different bronchi generations), mechanical percussion, highfrequency chest compression and non-invasive ventilation (NIV). Notably, PEP therapy can be oscillating or non oscillating. Oscillating PEP combines oscillation of airflow with PEP in order to loosen secretions [7,8]. Aerobic exercise can also be considered as an airway clearance technique, as it reduces mechanical impedance of sputum and enhances expiratory flow rates [9].

\section{Exercise training}

In pulmonary rehabilitation, out-patient, in-patient and homebased programs can be utilized. Exercise is a basic component of rehabilitation programs, and it includes lower and upper extremity training, inspiratory muscle training, as well as chest physical therapy techniques. Usually, the proposed schedule consists of a minimum of two sessions per week, preferably three or more, for 4, 6, 9 or 12 weeks (minimum of 12 supervised sessions recommended). The duration varies from 30 to $60 \mathrm{~min}$ and exercise can be continuous or intermittent and training can target endurance and/or strength. Patients should be able to continue effective training once supervised sessions have ended and regular physical activity five times a week for $30 \mathrm{~min}$ each time is encouraged regardless of rehabilitation, as healthy living advice $[10,11]$.

Notably, the American College of Sports Medicine recommend moderate-intensity aerobic (endurance) physical activity for a minimum of $30 \mathrm{~min}$ on five days each week or vigorous-intensity aerobic physical activity for a minimum of $20 \mathrm{~min}$ on three days each week in order to promote and maintain health in adults [12].

As far as home-based programs are concerned, several protocols have been used, based on aerobic and muscle strength training. Happ et al. utilized an at-home bicycle exercise regimen performed three times a week, consisting of two exercise routines of different intensity performed in an alternating sequence during each session. Schneiderman-Walker et al. also used a three times weekly program that required a minimum of $20 \mathrm{~min}$ of aerobic exercise at a heart rate of approximately 150 beats/min. While there is still no standardization regarding self-regulated, home-based exercise, further evaluation is needed as such interventions are fairly easily conducted and low-cost [13-15].

Kriemler et al. compared the effects of 6 months training (aerobic or anaerobic) versus no training and found similar improvements in $\mathrm{FEV}_{1}, \mathrm{FVC}$ and exercise capacity. However, anaerobic training lowered static hyperinflation, while aerobic training did not. The authors assumed that improvements in FVC with strength training might be because of a decrease in hyperinflation in this group [16] .Based on these facts, aerobic and anaerobic training can be regarded as similarly effective.

A randomized control trial by Selvadurai et al. compared aerobic and resistance training in children with cystic fibrosis (CF) admitted to hospital with an intercurrent pulmonary infection with a control group. The study demonstrated that children who received aerobic training had significantly better peak aerobic capacity, activity levels, and quality of life than children who received the resistance training program. Children who received resistance training had better weight gain (total mass, as well as fat-free mass), lung function, and leg strength than children who received aerobic training. Therefore it is implied that a combination of aerobic and anaerobic training may be the optimal training modality for $\mathrm{CF}$ patients [17].

It is noteworthy that not all training modalities are suitable for all patients, as in the case of severe pulmonary impairment. Gruber et al. used an individualized training program with supplemental oxygen over 6 weeks in patients with severe disease and found comparable improvements in exercise capacity with conventional training in patients with less severe disease [18]. This study is in favor of individualized training interventions.

Concerning the intensity of training, most studies that managed to improve exercise capacity used intensities at about $50 \% \mathrm{VO}_{2} \max$ or $60-85 \%$ of maximum heart rate $[16,19-21]$. A study of Whitley et al assessed the immediate effects of different exercise intensities on pulmonary function and diffusion capacity. Moderate exercise improved diffusion, while vigorous exercise caused airflow restrictions [22]. Taking all these into account, the optimal exercise training protocol for $\mathrm{CF}$ patients is yet to be defined.

\section{Education, behavioural change promotion and adjunctive therapies}

In addition to these, in pulmonary rehabilitation programs psychosocial assessment and intervention are offered, as depression and anxiety are common in patients with chronic lung disease. Evaluation of disability and education of both patient and family 
are part of this type of intervention [11]. Moreover, nutritional interventions are appropriate, considering the high probability of malnourishment and decreased muscle mass among patients with chronic respiratory conditions [11]. Adjunctive therapies, such as bronchodilators, oxygen therapy, non-invasive ventilation (NIV) and neuromuscular stimulation are also of considerable help during a rehabilitation program. Last but not least, patients referred to pulmonary rehabilitation should be assessed about their smoking status and smoking cessation services should be available [11].

\section{Integration of new technologies in pulmonary rehabilitation programs}

It is noteworthy that for the last couple of years, there has been special interest in new technologies, including video games, social media and web-based platforms and the potential for their application in the rehabilitation process.

In the CF population, a randomized controlled trial used the Nintendo Wii platform to deliver a 6-week home training program, with participants followed up for 12 months after the intervention. Exercise capacity, muscular strength and quality of life were improved in the short-term. The effects of training on muscle performance and quality of life were sustained over 12 months. However, adherence was $95 \%$ at 6 weeks but substantially reduced in the long term, with $65 \%$ of the subjects not using the active video game at all at 12 months [23]. Bishay et al. compared a fitness tracker with a personalized exercise prescription and social media platform to exercise prescription alone, although no significant difference in patient outcomes was noted between the two groups [24].

An RCT by Salonini et al. involving children and adolescents with CF compared a traditional stationary cycle training intervention to a training intervention using the interactive Xbox Kinect platform. Heart rate, $\mathrm{SpO}_{2}$, dyspnea, fatigue and subject satisfaction were evaluated. The study concluded that training with Xbox Kinect provided a cardiovascular demand similar to a stationary cycle, while it caused less dyspnea and fatigue and was more enjoyable than the stationary cycle. Therefore, such a modality has the potential to be used as an exercise intervention in young patients with CF. Similarly, Holmes et al. reported that training using the Xbox Kinect may be a suitable alternative to conventional exercise modalities for adults with CF $[25,26]$. O'Donovan et al. concluded that active video games are a useful source of light to moderate intensity physical activity in children with cystic fibrosis [27]. There are also other examples of studies including social media or webbased platforms as an aid to exercise $[28,29]$ and several relative studies that are on-going [30,31]

These new modalities have potentially beneficial effects on fitness and training adherence, however further research is needed to study the long-term effects of such interventions [32].

\section{What are the indications and contraindications of pulmonary rehabilitation?}

Presence of long-term debilitating symptoms, dyspnea Medical Research Council (MRC) score of more than 2, motivation of patient to follow the rehabilitation program, rehabilitation prior to volume reduction surgery or lung transplantation are some of the criteria for patient selection for pulmonary rehabilitation in general. However, rehabilitation is not usually appropriate when the patient is considered unable to follow the proposed program or when serious comorbidities are present, such as angina pectoris, recent myocardial infarction, severe pulmonary hypertension, unstable diabetes, severe exercise-induced hypoxemia, abdominal aortic aneurysm $>5.5 \mathrm{~cm}$ deemed inoperable, severe locomotor impairment, severe peripheral vascular disease. Stable cardiovascular disease is not a contraindication. Active cigarette smoking is, in certain cases, considered as a relative contraindication, although patients with COPD should be referred regardless of their smoking status $[10,11]$.

As far as CF patients are concerned, pulmonary rehabilitation is a key element of care. Airway clearance techniques are frequently described as a cornerstone of CF treatment and should be performed across the lifespan. Exercise is also recommended, and patients should be offered an individualized program, according to their capability and preferences. Regular physical activity should be encouraged and should include weightbearing exercise in order to optimize bone density, while strength training programs should be prescribed to optimize muscle mass. Nutritional and psychological interventions are also acknowledged as an integral part of the standard of care in $\mathrm{CF}[7,33]$.

Even in complex CF cases, physiotherapy management is advised to be continued, with proper alterations and modifications, rather than discontinued, when possible. For instance, in case of hemoptysis the regimens are altered to minimize the risk of rebleeding and in case of pneumothorax physiotherapy is continued when feasible but, minimizing the amount of positive pressure generated inside the patient lungs [34]. The American Diabetes Association Clinical Care Guidelines for CF-related diabetes recommend that patients should perform moderate aerobic exercise for at least 150 min per week, but monitoring of blood glucose levels before activity, consumption of extra carbohydrates or alterations of insulin dosage may be required [35].

Pregnant women with $\mathrm{CF}$ are encouraged to maintain a regular airway clearance routine, though postural drainage should be avoided. Exercise programs should be appropriate for the cardiorespiratory and musculoskeletal changes during pregnancy, and adequate hydration during exercise should be maintained [36]

\section{What are the benefits of pulmonary rehabilitation in general?}

Pulmonary rehabilitation is known to improve exercise capacity, muscle strength, dyspnea and health status compared with usual care. Self-reported measures of activities of daily living (ADL) and psychological status also improve. In addition to these, pulmonary rehabilitation modestly ameliorates physical activity levels and body weight/nutritional status [11].

\section{What are the reasons for reduced exercise capacity in patients with cystic fibrosis?}

Thick bronchial secretions, altered respiratory mechanics and gas exchange and decreased lung function are hallmark features of the disease. Severe airflow limitation and dynamic hyperinflation results in increased dead space ventilation and exertional dyspnea. In certain patients, concomitant cardiovascular abnormalities may play a role. Another important factor in the impairment to exercise is the involvement of peripheral muscles. Defective muscle metabolism, malnutrition, electrolyte disturbances, physical 
inactivity, systemic inflammation and oxidative stress, oral corticosteroid use and CF-specific gene defects can be implicated in the peripheral muscle dysfunction. In certain cases, diaphragm strength is decreased despite a normal muscle mass, which can be possibly attributed to functional changes due to hyperinflation [37].

\section{What are the benefits of airway clearance techniques in CF patients?}

Concerning airway clearance, bibliography clearly recommends its application when compared to no airway clearance or cough alone [11,38-40]. A 2015 Cochrane review found a significant increase in the amount of sputum expectorated in the patient groups that applied airway clearance compared to spontaneous cough or not using any airway clearance technique, concluding that methods of clearing the airways have short-term benefits for moving mucous [39]. Most studies do not show significant differences in the lung function of CF patients after chest physiotherapy [8,39,41-43]. Evidence from the Cochrane systematic reviews support that no one airway clearance regimen is better than another [8,31].

\section{What are the benefits of exercise training in $\mathrm{CF}$ patients?}

Evidence suggests that $\mathrm{CF}$ patients with better physical fitness have better quality of life [7]. Exercise programs can improve fitness, exercise capacity, thoracic mobility, quality of life, maintain bone mineral density and lower the rate of decline in pulmonary function [13,19,44-51]. Moreover, a training effect, as measured by a decrease in lactate levels and heart rate can be achieved [52]. Both aerobic or anaerobic physical training has a positive effect on primary outcomes in CF patients (exercise capacity, strength and lung function) [53].

\section{Pulmonary function and exercise capacity}

A prospective, longitudinal study by Schneiderman et al. that included 212 patients with CF recruited over a 9-year period, concluded that patients with CF with increasing activity levels had a reduced rate of $\mathrm{FEV}_{1}$ decline compared to those who did not become more active over the study period [54]. Kriemler et al. and Hebestreit et al. both studied the effects of physical training over a 6-month period and found improved exercise capacity and lung function in the intervention group compared to the control group [16,55]. Santana-Sosa et al. reported amelioration of muscle strength and $\mathrm{VO}_{2}$ peak after an 8-week exercise program [20,21]. A retrospective cohort study of 2014 concluded that regular exercise is associated with a reduced decline in $\mathrm{FEV}_{1}$ and body mass index (BMI) in adults with CF. It is noteworthy that exercise capacity seems to be linked to the prognosis of $\mathrm{CF}$ patients. $\mathrm{VO}_{2}$ peak, peak work rate, ventilatory equivalent for oxygen and carbon dioxide have been found as predictors of death or lung transplantation at 10year follow-up [56].

\section{Health-related quality of life, need for hospitalization, body posture and strength}

$\mathrm{VO}_{2}$ peak correlates with HRQL in CF patients and when improved it is associated with better nutritional status and health perception [57]. Hebestreit et al. concluded that a combined aerobic and strength training ameliorates exercise capacity, lung function and HRQL [19]. Another RCT by Klijn et al. has shown that anaerobic training has a beneficial effect on aerobic and anaerobic performance, as well as on quality of life [50].

Urquhart et al. reported that supervised, outpatient exercise and physiotherapy are associated with improvements in QOL and exercise tolerance, a reduction in intravenous antibiotic days, and a trend towards reducing lung function decline in children with $\mathrm{CF}$ [58]. Perez et al. detected that $\mathrm{VO}_{2}$ peak was the only variable significantly associated with time to hospitalization, therefore greater aerobic fitness is associated with a lower risk of hospitalization [59].

An RCT by Schindel et al. studied the effect of exercise training on posture, and resulted that after 3 months the intervention group showed a decrease in cervical and lumbar lordosis, thoracic kyphosis, lateral chest distance and abdominal protrusion [60]. Rovedder et al. reported increased upper limb strength over a 3month combined aerobic and strength training program [14].

\section{Are there risks related to exercise? And if so, do the benefits outweigh the risks in the CF population?}

A patient may experience dyspnea, productive cough and fatigue during exercise. Moreover, some patients with bronchiectasis or asthma may suffer exercise-induced bronchoconstriction. Exercise-induced hypoxemia (drop in oxygen saturation by more than 4 or below $90 \%$ ) may also be observed, especially in patients with advanced lung disease. An estimated 20$25 \%$ of all patients with CF may present exercise-induced hypoxemia [36]. Patients with exercise-induced hypoxemia, as well as patients treated with macrolides are also at higher risk of developing cardiac arrhythmias during exercise. In general, approximately $5-10 \%$ of all patients present cardiac arrhythmias with exercise. Furthermore, exercise can potentially trigger a pneumothorax or an episode of hemoptysis, although the evidence is scarce. Musculoskeletal injuries are always possible with exercise, just as in the healthy population [61].

As far as CF patients are concerned, electrolyte losses, injury to spleen or oesophageal hemorrhage in the presence of portal hypertension, hypoglycemia in the setting of CF-related diabetes mellitus and worsening of preexisting arthritis are potential risks during exercise. In addition to these, when pulmonary rehabilitation programs are organized, special attention should be given to hygiene aspects to avoid cross-infection.

The number of different complications described may be unsettling, however the frequency of true adverse events is small and these mainly appear in patients with advanced disease. Individual assessment of each patient is always advised, but in most cases the benefits of exercise clearly outweigh the potential harms [61-63].

\section{Discussion}

Pulmonary rehabilitation is a key component in CF care. Traditionally, airway clearance techniques are considered a cornerstone of CF treatment and should be performed across the lifespan in CF [7]. Evidence supports that airway clearance 
regimens have beneficial effects for moving mucous and are similarly effective $[8,39]$.

Regular exercise training is increasingly being recommended for $\mathrm{CF}$ patients. Further research is necessary to accurately assess the benefits of exercise training in the $\mathrm{CF}$ population. Moreover, there is a lack of studies investigating the effects of physical exercise training on other significant outcomes, such as bone health, diabetic control and pulmonary exacerbations. However, evidence for the beneficial effects of exercise training on lung health and exercise capacity is growing. Exercise improves aerobic capacity and probably lowers the rate of decline in pulmonary function. Exercise capacity is also correlated with the prognosis of CF patients [56]. Thus, there is no reason to discourage the integration of exercise in all CF patients care [32]. This is also supported by evidence that show a relatively small frequency of true adverse events of exercise. Personalized assessment of patients is needed, but in most cases the benefits of exercise outweigh the potential harms [61,64].

The optimal training components (type, frequency, duration, intensity) need to be determined. Taking into account the existing literature, exercise training programs need to be tailored to the patient. For non-complex cases, perhaps a program of moderateintensity exercise, 5 times per week, for $30 \mathrm{~min}$ per session is a reasonable regimen [12,19-22,65]. There is an increasing interest in the integration of new technologies in the pulmonary rehabilitation process (video game activities, social media and web-based platforms). These new modalities have potentially beneficial effects on fitness and training adherence, however further research is needed to study the long-term effects of such interventions [32].

\section{Limitations}

This article is a narrative review, therefore it represents a broad overview of a topic-related research area. Data extraction relies on description of study findings rather than on continuous or categorical statistical values.

\section{Conclusions}

Pulmonary rehabilitation is a multidisciplinary, individualized intervention that incorporates exercise training, airway clearance, education and behaviour change and a key component in $\mathrm{CF}$ patients care, as it can improve exercise capacity, muscle strength, quality of life and nutritional status of patients.

Both airway clearance techniques and exercise training are invaluable parts of pulmonary rehabilitation. Airway clearance regimens have beneficial effects for moving mucous, and they seem to be similarly effective. On the other hand, exercise improves aerobic capacity (commonly affected in CF due to multifactorial pathology) and probably lowers the rate of decline in pulmonary function in $\mathrm{CF}$ patients. The optimal training components (type, frequency, duration, intensity) need to be determined, but it seems that a combination of aerobic and anaerobic training may be the optimal training modality for $\mathrm{CF}$ patients. When planning a training program, personalized assessment is needed, but in most cases the benefits of exercise outweigh the potential harms, so there is no reason to discourage the integration of exercise in all CF patients care.

In the past few years, new technologies (video game activities, social media and web-based platforms) can also be integrated in the rehabilitation process. Further research is needed to study the long- term effects of such interventions, however new means offer new possibilities for CF care. For instance, tele-rehab could be evolved and become a safe standard of care, especially during the COVID19 era.

\section{References}

1. Hollin IL, Robinson KA. A Scoping review of healthcare costs for patients with cystic fibrosis. Appl Health Econ Health Policy 2016;14:151-9.

2. Davies JC, Alton EW, Bush A. Cystic fibrosis. BMJ 2007;335:1255-9.

3. Spruit MA. Pulmonary rehabilitation. Eur Respir Rev 2014;23:55-63.

4. Spinou A. Physiotherapy in cystic fibrosis A comprehensive clinical overview. Pneumon 2018;31:35-43.5.

5. PRISMA. Transparent reporting of systematic reviews and meta-analyses.

6. McIlwaine M, Bradley J, Elborn JS, Moran F. Personalising airway clearance in chronic lung disease. Eur Respir Rev 2017;26:160086. Available from: http://www.prisma-statement.org/

7. Button BM, Wilson C, Dentice R, et al. Physiotherapy for cystic fibrosis in Australia and New Zealand: A clinical practice guideline. Respirology 2016;21:656-67.

8. Bradley JM, Moran FM, Elborn JS. Evidence for physical therapies (airway clearance and physical training) in cystic fibrosis: an overview of five Cochrane systematic reviews. Respir Med 2006;100:191-201

9. Dwyer TJ, Alison JA, McKeough ZJ, et al. Effects of exercise on respiratory flow and sputum properties in patients with cystic fibrosis. Chest 2011;139:870-7.

10. Sharma BB, Singh V. Pulmonary rehabilitation: An overview. Lung India 2011;28:276-84.

11. Bolton CE, Bevan-Smith EF, Blakey JD, et al. British Thoracic Society guideline on pulmonary rehabilitation in adults. Thorax 2013;68:ii1-30.

12. Haskell WL, Lee IM, Pate RR, et al. Physical activity and public health: updated recommendation for adults from the American College of Sports Medicine and the American Heart Association. Med Sci Sports Exerc 2007;39:1423-34.

13. Schneiderman-Walker J, Pollock SL, Corey M, et al. A randomized controlled trial of a 3-year home exercise program in cystic fibrosis. J Pediatr 2000;136:304-10.

14. Rovedder PM, Flores J, Ziegler B, et al. Exercise programme in patients with cystic fibrosis: a randomized controlled trial. Respir Med 2014;108:1134-40.

15. Happ MB, Hoffman LA, Higgins LW, et al. Parent and child perceptions of a self-regulated, home-based exercise program for children with cystic fibrosis. Nurs Res 2013;62:305-14. Erratum in: Nurs Res 2014;63:25. DiVirgilio, Dana [corrected to Divirgilio, Dana].

16. Kriemler S, Kieser S, Junge S, et al. Effect of supervised training on FEV1 in cystic fibrosis: a randomised controlled trial. J Cyst Fibros 2013;12:714-20.

17. Selvadurai HC, Blimkie CJ, Meyers N, et al. Randomized controlled study of in-hospital exercise training programs in children with cystic fibrosis. Pediatr Pulmonol 2002;33:194-200.

18. Gruber W, Orenstein DM, Braumann KM, Beneke R. Interval exercise training in cystic fibrosis -- effects on exercise capacity in severely affected adults. J Cyst Fibros 2014;13:86-91. 
19. Hebestreit H, Kieser S, Junge S, et al. Long-term effects of a partially supervised conditioning programme in cystic fibrosis. Eur Respir J 2010;35:578-83.

20. Santana-Sosa E, Gonzalez-Saiz L, Groeneveld IF, et al. Benefits of combining inspiratory muscle with 'whole muscle' training in children with cystic fibrosis: a randomised controlled trial. Br J Sports Med 2014;48:1513-7.

21. Sosa ES, Groeneveld IF, Gonzalez-Saiz L, et al. Intrahospital weight and aerobic training in children with cystic fibrosis: A randomized controlled trial. Med Sci Sports Exerc 2012; $44: 2-11$

22. Wheatley CM, Baker SE, Morgan MA, et al. Effects of exercise intensity compared to albuterol in individuals with cystic fibrosis. Respir Med 2015;109:463-74.

23. Del Corral T, Cebrià I Iranzo MÀ, et al. Effectiveness of a home-based active video game programme in young cystic fibrosis patients. Respiration 2018;95:87-97.

24. Bishay LC, Nelson E, Williams K, et al. Effect of a wearable fitness tracker on exercise tolerance for adults with cystic fibrosis: a pilot randomized clinical trial. Pediatr Pulmonol 2018;53(S2):345. Added to CENTRAL: 30 November 2018 | 2018 Issue 11.

25. Holmes H, Wood J, Jenkins S, et al. Xbox Kinect ${ }^{\mathrm{TM}}$ represents high intensity exercise for adults with cystic fibrosis. J Cyst Fibros 2013;12:604-8

26. Salonini E, Gambazza S, Meneghelli I, et al. Active video game playing in children and adolescents with cystic fibrosis: Exercise or just fun? Respir Care 2015;60:1172-9.

27. O'Donovan C, Greally P, Canny G, et al. Active video games as an exercise tool for children with cystic fibrosis. J Cyst Fibros 2014;13:341-6.

28. Smith A, Gouick L. WS05.2 30 day challenge - using social media to support adult $\mathrm{CF}$ patients to exercise in the adult $\mathrm{CF}$ service Dundee. J Cyst Fibros 2015;14;S9.

29. Cox NS, Alison JA, Button BM, et al. Feasibility and acceptability of an internet-based program to promote physical activity in adults with cystic fibrosis. Respir Care 2015;60:422-9.

30. Cox NS, Eldridge B, Rawlings S, et al. A web-based intervention to promote physical activity in adolescents and young adults with cystic fibrosis: protocol for a randomized controlled trial. BMC Pulm Med 2019;19:253.

31. Lang RL, Wilson C, Stockton K, et al. CyFiT telehealth: protocol for a randomised controlled trial of an online outpatient physiotherapy service for children with cystic fibrosis. BMC Pulm Med 2019;19:21.

32. Hebestreit H, Kriemler S, Radtke T. Exercise for all cystic fibrosis patients: is the evidence strengthening? Curr Opin Pulm Med 2015;21:591-5.

33. National Guideline Alliance (UK). Cystic Fibrosis: Diagnosis and management. London: National Institute for Health and Care Excellence (UK); 2017 Oct 25.

34. Flume PA, Mogayzel PJ Jr, Robinson KA, et al. Cystic fibrosis pulmonary guidelines: pulmonary complications: hemoptysis and pneumothorax. Am J Respir Crit Care Med 2010;182:298-306.

35. Moran A, Brunzell C, Cohen RC, et al. Clinical care guidelines for cystic fibrosis-related diabetes: a position statement of the American Diabetes Association and a clinical practice guideline of the Cystic Fibrosis Foundation, endorsed by the Pediatric Endocrine Society. Diabetes Care 2010;33:2697-708.

36. Johannesson M. Effects of pregnancy on health: certain aspects of importance for women with cystic fibrosis. J Cyst Fibros 2002;1:9-12.
37. Burtin $\mathrm{C}$, Hebestreit $\mathrm{H}$. Rehabilitation in patients with chronic respiratory disease other than chronic obstructive pulmonary disease: exercise and physical activity interventions in cystic fibrosis and non-cystic fibrosis bronchiectasis. Respiration 2015;89:181-9.

38. Bott J, Blumenthal S, Buxton M, et al. Guidelines for the physiotherapy management of the adult, medical, spontaneously breathing patient. Thorax 2009;64:i1-51.

39. Warnock L, Gates A. Chest physiotherapy compared to no chest physiotherapy for cystic fibrosis. Cochrane Database Syst Rev 2015;2015:CD001401.

40. Main E, Prasad A, Schans C. Conventional chest physiotherapy compared to other airway clearance techniques for cystic fibrosis. Cochrane Database Syst Rev 2005:CD002011.

41. Morrison L, Agnew J. Oscillating devices for airway clearance in people with cystic fibrosis. Cochrane Database Syst Rev 2014:CD006842.

42. Elkins MR, Jones A, van der Schans C. Positive expiratory pressure physiotherapy for airway clearance in people with cystic fibrosis. Cochrane Database Syst Rev 2006:CD003147.

43. Mckoy NA, Wilson LM, Saldanha IJ, et al. Active cycle of breathing technique for cystic fibrosis. Cochrane Database Syst Rev 2016;7:CD007862.

44. Bradley J, Moran F. Physical training for cystic fibrosis. Cochrane Database Syst Rev 2008;1:CD002768.

45. Elbasan B, Tunali N, Duzgun I, Ozcelik U. Effects of chest physiotherapy and aerobic exercise training on physical fitness in young children with cystic fibrosis. Ital J Pediatr 2012;38:2.

46. Hulzebos HJ, Snieder H, van der Et J, et al. High-intensity interval training in an adolescent with cystic fibrosis: a physiological perspective. Physiother Theory Pract 2011;27:231-7.

47. Shoemaker MJ, Hurt H, Arndt L. The evidence regarding exercise training in the management of cystic fibrosis: a systematic review. Cardiopulm Phys Ther J 2008;19:75-83.

48. Tejero García S, Giráldez Sánchez MA, Cejudo P, et al. Bone health, daily physical activity, and exercise tolerance in patients with cystic fibrosis. Chest 2011;140:475-81.

49. Paranjape SM, Barnes LA, Carson KA, et al. Exercise improves lung function and habitual activity in children with cystic fibrosis. J Cyst Fibros 2012;11:18-23.

50. Klijn PH, Oudshoorn A, van der Ent CK, et al. Effects of anaerobic training in children with cystic fibrosis: a randomized controlled study. Chest 2004;125:1299-305.

51. Schmidt AM, Jacobsen U, Bregnballe V, et al. Exercise and quality of life in patients with cystic fibrosis: A 12-week intervention study. Physiother Theory Pract 2011;27:548-56.

52. Moorcroft AJ, Dodd ME, Morris J, Webb AK. Individualised unsupervised exercise training in adults with cystic fibrosis: a 1 year randomised controlled trial. Thorax 2004;59:1074-80.

53. Bradley J, Moran F. Physical training for cystic fibrosis. Cochrane Database Syst Rev. 2008;1:CD002768.

54. Schneiderman JE, Wilkes DL, Atenafu EG, et al. Longitudinal relationship between physical activity and lung health in patients with cystic fibrosis. Eur Respir J 2014;43:817-23.

55. Hebestreit H, Kieser S, Junge S, et al. Long-term effects of a partially supervised conditioning programme in cystic fibrosis. Eur Respir J 2010;35:578-83.

56. Hebestreit H, Hulzebos EHJ, Schneiderman JE, et al. Cardiopulmonary exercise testing provides additional prognostic information in cystic fibrosis. Am J Respir Crit Care Med 2019;199:987-95.. Erratum in: Am J Respir Crit Care Med 2020;202:780.

57. Hebestreit H, Schmid K, Kieser S, et al. Quality of life is asso- 
ciated with physical activity and fitness in cystic fibrosis. BMC Pulm Med 2014;14:26.

58. Urquhart D, Sell Z, Dhouieb E, et al. Effects of a supervised, outpatient exercise and physiotherapy programme in children with cystic fibrosis. Pediatr Pulmonol 2012;47:1235-41.

59. Pérez M, Groeneveld IF, Santana-Sosa E, et al. Aerobic fitness is associated with lower risk of hospitalization in children with cystic fibrosis. Pediatr Pulmonol 2014;49:641-9.

60. Schindel CS, Hommerding PX, Melo DA, et al. Physical exercise recommendations improve postural changes found in children and adolescents with cystic fibrosis: a randomized controlled trial. J Pediatr 2015;166:710-6.e2.

61. Burtin C, Hebestreit H. Rehabilitation in patients with chronic respiratory disease other than chronic obstructive pulmonary disease: exercise and physical activity interventions in cystic fibrosis and non-cystic fibrosis bronchiectasis. Respiration 2015;89:181-9.

62. Lebecque P, Lapierre JG, Lamarre A, Coates AL. Diffusion capacity and oxygen desaturation effects on exercise in patients with cystic fibrosis. Chest 1987;91:693-7.

63. Albert RK, Schuller JL, COPD Clinical Research Network. Macrolide antibiotics and the risk of cardiac arrhythmias. Am J Respir Crit Care Med 2014;189:1173-80.

64. Radtke T, Nevitt SJ, Hebestreit H, Kriemler S. Physical exercise training for cystic fibrosis. Cochrane Database Syst Rev 2017;11:CD002768.

65. Kriemler S, Kieser S, Junge S, et al. Effect of supervised training on FEV1 in cystic fibrosis: a randomised controlled trial. J Cyst Fibros 2013;12:714-20. 